\title{
Rutherford and Geiger Re-evaluated
}

ADVANCES in nuclear physics in the past decade have arisen chiefly from developments in instrumentation coupled with the higher energy accelerators that became available in the 1960s. It is more than possible that the suggestion by Dr Peter Rice-Evans on page 625 of this issue of Nature will eventually be developed into a technique that will have universal application, although the theoretical predictions have yet to be tested. There are certain to be many Jeremiahs who will deem the idea for a new high energy particle detector as unpractical, but there will be few who will argue that the idea is not worth testing.

Rice-Evans's suggestion is based on the proportional counter concept first developed by Rutherford and Geiger in 1908. The novelty of the present approach is that the proportional counter be used in reverse with the outer cylinder being held at a positive potential with respect to the centre wire. The electrons liberated by the passage of an ionizing particle will thus be accelerated towards the outer cylinder and not towards the centre as in a conventional proportional counter. In this way a pulse with a much faster rise can be expected and the instrument becomes, in principle, a powerful tool that can be used to determine the position of an ionizing particle with a higher degree of precision than is currently possible.

To appreciate the advantages to high energy physics if this detector ever became a practical proposition it only needs to be realized that there is a great deal of current concern that the resolutions currently available with wide plane spark chambers, streamer chambers and multi-wire proportional counters will not be adequate to meet the requirements of the experiments that will be carried out with the new generation of synchrotrons typified by the one being built at Batavia in Illinois, and the $300 \mathrm{GeV}$ machine planned for CERN. The higher energy of the accelerated beam means that the analysed particles will also have a higher energy and will thus suffer smaller deflexions in analysing magnets than particles accelerated in machines of lower energies. There is a need therefore for detectors with good spatial resolution and none of the detectors in current use meets the requirements.

How does the proposed reverse proportional counter supply the necessary high resolution? In such a set up the avalanche produced by an ionizing particle entering the chamber will be outwardly directed, and pulses will only arise in the wire when electrons are liberated near the wire surface in the region of high field necessary for avalanche growth. This is to be compared with a conventional proportional counter where a particle is detected irrespective of where in the chamber the event occurs. The difference emphasizes that the proportional counter was initially designed to maximize the efficiency rather than the spatial resolution as in the present proposed use. The analysis of Rice-Evans shows that a pulse produced by an ion within the critical distance needed to form the avalanche achieves 22 per cent of its maximum height within $10^{10} \mathrm{~s}$ of the event occurring. This fast rise is ideally suited for obtaining a fast pulse from a differentiation network to trigger the electronic circuits that are necessary in high energy physics experiments. Thus to obtain a pulse from the chamber an electron must be formed near the wire surface within the critical avalanche radius. It is here that the crucial question of the efficiency of the proposed device arises. To obtain maximum efficiency the radius of the wire needs to be as large as possible, but large wire radius is incompatible with the good spatial resolution that the detector sets out to achieve in the first place. Thus the ever present problem of achieving a compromise between efficiency and resolution would seem to limit the usefulness of the detector.

Calculations by Rice-Evans show that for a typical chamber the region of interaction is limited to a cylinder about the central wire of radius approximately twice that of the wire itself. The efficiency of producing an electron within this region depends on whether the interaction occurs in the wire or in the gas of the chamber, and the probability for these processes is estimated to differ by an order of magnitude-the interaction in the wire being most likely to produce an electron. Thus, the detector radius is effectively the same as the wire radius, and to achieve both high efficiency and high resolution a matrix of many wires will be needed. Such systems are already in use with multi-wire proportional counters.

It is now time to carry out an experimental study with the reverse proportional counter. The problems to be overcome are certain to be many, but only time will tell whether they are insurmountable. Multi-wire proportional counters were first used in 1956, but have only recently achieved universal usage. If the suggested detector is to be used with the new generation of high energy accelerators, however, it has to be developed at a faster pace. The decisions to be made before starting experimental work are many and involve such fundamentals as the material to be used for the wire, what gas to be used in the chamber-or whether a noble liquid could be used instead. The dimensions of the chamber have also to be detected, but of more fundamental importance is the question of how spurious positive ions might affect the performance. Such ions might cause ionization of the gas in the high field region or they might cause secondary electrons to be freed from the surface of the wire. A possible way to circumvent these problems would be to introduce a vapour to neutralize the spurious gas ions and so quench the discharge.

The possibility of the detector being a viable proposition depends entirely on how the expected one to several thousand signal to noise ratio that will arise from particles forming ions outside the avalanche region can be overcome. For a chamber of the dimensions given by Rice-Evans the ratio is one to 5,000 as only 1.5 per cent of the particles will pass through the avalanche region.

The chances of a successful detector being developed in the face of all these uncertainties must be rather small, but several innovations that have in the past seemed to the scientists of the day to have even less chances of developing into viable entities are now accepted techniques. Such a detector or series of detectors must be built and tested. 